\title{
Genome-wide linkage disequilibrium and genetic diversity in five populations of Australian domestic sheep
}

\author{
Hawlader Abdullah Al-Mamun', Samuel A Clark', Paul Kwan² and Cedric Gondro ${ }^{\text {** }}$
}

\begin{abstract}
Background: Knowledge of the genetic structure and overall diversity of livestock species is important to maximise the potential of genome-wide association studies and genomic prediction. Commonly used measures such as linkage disequilibrium (LD), effective population size $\left(N_{e}\right)$, heterozygosity, fixation index $\left(F_{S T}\right)$ and runs of homozygosity $(\mathrm{ROH})$ are widely used and help to improve our knowledge about genetic diversity in animal populations. The development of high-density single nucleotide polymorphism (SNP) arrays and the subsequent genotyping of large numbers of animals have greatly increased the accuracy of these population-based estimates.
\end{abstract}

Methods: In this study, we used the Illumina OvineSNP50 BeadChip array to estimate and compare LD (measured by $r^{2}$ and $\left.D^{\prime}\right), N_{e}$ heterozygosity, $F_{S T}$ and $\mathrm{ROH}$ in five Australian sheep populations: three pure breeds, i.e., Merino (MER), Border Leicester (BL), Poll Dorset (PD) and two crossbred populations i.e. F1 crosses of Merino and Border Leicester $(\mathrm{M} \times \mathrm{B})$ and $\mathrm{M} \times \mathrm{B}$ crossed to Poll Dorset (MxBxP).

Results: Compared to other livestock species, the sheep populations that were analysed in this study had low levels of $L D$ and high levels of genetic diversity. The rate of $L D$ decay was greater in Merino than in the other pure breeds. Over short distances ( $<10 \mathrm{~kb}$ ), the levels of LD were higher in BL and PD than in MER. Similarly, BL and PD had comparatively smaller $N_{e}$ than MER. Observed heterozygosity in the pure breeds ranged from 0.3 in BL to 0.38 in MER. Genetic distances between breeds were modest compared to other livestock species (highest $F_{\mathrm{ST}}=0.063$ ) but the genetic diversity within breeds was high. Based on $\mathrm{ROH}$, two chromosomal regions showed evidence of strong recent selection.

Conclusions: This study shows that there is a large range of genome diversity in Australian sheep breeds, especially in Merino sheep. The observed range of diversity will influence the design of genome-wide association studies and the results that can be obtained from them. This knowledge will also be useful to design reference populations for genomic prediction of breeding values in sheep.

\section{Background}

The process of sheep domestication began between 9000 and 11,000 years ago. Over thousands of years, humans have selected sheep for different desirable production traits such as wool, milk and meat. This artificial selection combined with natural adaptation to new environments as sheep were introduced throughout the world has led

\footnotetext{
*Correspondence: cgondro2@une.edu.au

1 School of Environmental and Rural Science, University of New England,

Armidale, NSW 2351, Australia

Full list of author information is available at the end of the article
}

to a broad spectrum of phenotypic diversity with more than one thousand different sheep breeds [1]. Australia is one of the world's largest sheep producers for wool and meat, with the Merino breed being the most economically important. Merino animals account for approximately $75 \%$ of all Australian sheep (Year book Australia 2003, Australian Bureau of Statistics) and another $12 \%$ of the population are Merino $x$ Border Leicester F1 crosses that produce high-quality females used for meat production. These animals are commonly crossed with meat breeds such as Poll Dorset for the production of prime 
lambs (http://www.polldorset.org.au). The three pure breeds Merino, Poll Dorset, Border Leicester and their crosses (Merino $\times$ Border Leicester and Merino $\times$ Border Leicester $\times$ Poll Dorset) represent more than $90 \%$ of the Australian sheep population.

Understanding the genetic diversity of these key sheep breeds in Australia is important to design and interpret studies that investigate genome-wide association and genomic prediction. For example, the amount of diversity in a population is a key indicator of the amount of phenotypic data (reference data) that is required to obtain accurate genomic predictions. This is also important when interpreting genome-wide association studies (GWAS) data since high levels of diversity reduce the likelihood that highly significant markers are at a large distance from the quantitative trait locus (QTL) that underlies variation in phenotype and allows for easier identification of possible functional regions.

Genetic diversity can be estimated from pedigree data or from molecular marker data. Estimates of genetic diversity are more robust when marker data is used and particularly so when pedigree records are poor or shallow. However, this advantage is small or absent when low-density markers such as microsatellites are used [2-4]. The high-density single nucleotide polymorphism (SNP) arrays that are currently available have provided the opportunity to estimate genetic diversity parameters in livestock at a much higher level of definition than was previously possible.

There are a number of methods that can be used to estimate genetic diversity using marker data. These include observed and expected heterozygosity [5, 6], runs of homozygosity $(\mathrm{ROH})$ [7], Wright's $F$ statistic $\left(F_{\mathrm{ST}}\right)$ [8], linkage disequilibrium (LD) and effective population size $\left(N_{e}\right)$ [9].

Heterozygosity measures the genetic variation within a population and is one of the most widely used genetic diversity parameters [10]. A high level of heterozygosity indicates more genetic variability while a low level indicates little genetic variability and a small $N_{e}$. Wright's $F$ statistics $\left(F_{\mathrm{IT}}, F_{\mathrm{IS}}, F_{\mathrm{ST}}\right)$ are widely used to estimate genetic diversity within and between populations $[3,11]$. Runs of homozygosity are contiguous stretches of homozygous genotypes (e.g., an individual inherits the same haplotype from both parents). Long $\mathrm{ROH}$ could be a sign of recent inbreeding in a population whereas shorter $\mathrm{ROH}$ suggest loss of genetic diversity either from a population bottleneck or a founder effect (e.g., breed formation in livestock).

LD between any two markers reflects the extent of nonrandom association between them. LD underpins selection decisions in a wide range of livestock species that have adopted genetic technologies for selection purposes. Marker-assisted selection (MAS), genomic selection and GWAS all largely depend on the extent of LD within a population. It is the extent of LD that determines the minimum number of markers required for a successful genome-wide study; with LD remaining high over longer chromosomal segments, fewer markers are needed. Conversely, denser panels are required if LD decays rapidly. The pattern of LD decay also provides information on the evolutionary history of a population and can be used to estimate, e.g., the ancestral $N_{e}[12,13] . N_{e}$ size and other genetic events such as selection, migration, mutation and recombination events influence the extent of $L D$ within a population [14]. Comparison of the extent of LD between breeds is therefore informative about the overall diversity level in a species and can help us understand the patterns of selection that individual breeds have been subjected to. Due to its importance, various studies have reported LD estimates in various livestock species, e.g. cattle [1517], pig [18], horse [19], chicken [20] and sheep [21-23].

Our objective was to describe and compare LD patterns, and the level and structure of genetic diversity in the five commercial Australian sheep populations mentioned above. The results are expected to provide valuable information for the design and analysis of genetic association studies and genomic selection, as well as for the management of genetic resources in the most economically important Australian sheep populations.

\section{Methods}

\section{Ethics statement}

Samples for genotyping were collected under approval number 344 AEC12-049 of the University of New England Animal Ethics Committee.

\section{Animal resources}

The study consisted of 1273 sheep chosen from the Australian Sheep CRC Information Nucleus flock from five different populations: three pure breeds i.e., Border Leicester (BL; $n=253$ ), Merino (MER; $n=265$ ), Poll Dorset (PD; $n=264$ ), and two crossbred populations i.e., Merino and Border Leicester F1 crosses (MxB; $n=260)$, and crosses of Merino $\times$ Border Leicester with Poll Dorset (MxBxP; $n=231)$. MER is a wool breed, while BL and PD are primarily meat breeds; each breed was separately selected for its own specific purposes. $\mathrm{MxB}$ and $\mathrm{MxBxP}$ are straight $\mathrm{F} 1$ crosses from the pure breeds rather than terminal composite breeds and are not subjected to artificial selection. These crosses are often used for both wool and meat production. All animals were genotyped using the Illumina OvineSNP50 BeadChip (Illumina Inc., San Diego, CA, USA), which includes 54,241 SNPs. 


\section{Genotyping and quality control}

A number of quality control measures were applied to all SNPs as follows: SNPs were removed if they had a call rate $<95 \%$, a GC score $<0.6$, a minor allele frequency (MAF) $<0.01$ and if deviation in SNP heterozygosity was greater than three standard deviations from the mean. Data was also removed if SNP genotypes deviated from the Hardy-Weinberg equilibrium (for a P value cut-off of $1 \times 10^{-15}$ ) and had no assigned genomic location. Markers on sex chromosomes were also excluded from the analysis. Quality control was performed using snpQC [24]. Missing genotypes were imputed using BEAGLE 3 [25].

\section{SNP genome coordinates on the ovine genome sequence assembly}

Chromosomal coordinates for each SNP were obtained by aligning the region that covered approximately $120 \mathrm{bp}$ around each SNP, to the latest release of the ovine genome sequence assembly, Oar_v3.1, by BLAST (http:// blast.ncbi.nlm.nih.gov/Blast.cgi). Forty-one markers were excluded from the analysis because they had no assigned genomic location in Oar_v3.1.

\section{Genetic analysis of gene diversity}

Gene diversity or expected heterozygosity $\left(H_{E}\right)$ was calculated as a measure of genetic diversity. $H_{E}$ for each SNP was calculated separately and then averaged to find the average genetic diversity for each breed. Population relatedness was evaluated using pair-wise estimates of $F_{\mathrm{ST}}$. First, expected heterozygosities for subpopulations $(H S)$ and for the total population $(H T)$ were calculated for each locus for each pair of populations, then $H S$ and $H T$ were averaged across all loci. $H$ values (expected heterozygosities for populations and combined populations) were calculated as 1 minus the sum of the squared allele frequencies at a locus. To calculate $H T$, allele frequencies between two populations were averaged before the calculation. To calculate $H S, H$ values were averaged after the calculation. In both cases, the averages were weighted by the relative sizes of the two populations. $F_{S T}$ was then calculated for each pair of populations as $(H T-H S) / H T$. A principal component analysis (PCA) was used to examine the genetic structure of the five populations. The PCA was performed using a genomic relationship matrix [26] to define the covariance between animals. The relationship between the first two principal components was examined to show the relationship between the five populations.

\section{Measure of runs of homozygosity}

Runs of homozygosity were defined for individuals in each of the five populations using PLINK v1.07 [27] with sliding windows of $1000 \mathrm{~kb}$ across the genome to estimate homozygosity. A maximum of two SNPs with missing genotypes were allowed per window and up to one possible heterozygous genotype was permitted. To minimize the number of false positives, the minimum number of SNPs that constituted a $\mathrm{ROH}(l)$ was calculated by a method similar to that proposed by [28]:

$$
l=\frac{\log _{e} \frac{\alpha}{n_{s} \cdot n_{i}}}{\log _{e}(1-h e t)}
$$

where $n_{s}$ was the number of genotyped SNPs per individual, $n_{i}$ was the number of individuals, $\alpha$ was the percentage of false positive $\mathrm{ROH}$ (set to 0.05 in the present study) and het was the mean SNP heterozygosity across all SNPs. Since very short and common $\mathrm{ROH}$ are often due to $\mathrm{LD}, \mathrm{ROH}$ that were $<500 \mathrm{~kb}$ long were removed. Finally, the maximum gap between consecutive homozygous SNPs was set to $250 \mathrm{~kb}$.

Runs of homozygosity were estimated for each individual and then categorized based on $\mathrm{ROH}$ length (1-5 Mb, 5-10 Mb, 10-15 Mb, 15-20 Mb, 20-25 Mb, and $>25 \mathrm{Mb}$ ). The mean sum of $\mathrm{ROH}$ within each $\mathrm{ROH}$ category was calculated by adding up the length of all $\mathrm{ROH}$ for each individual in each $\mathrm{ROH}$ category and then the results were averaged per breed population. The percentage of occurrences of a SNP in a ROH was calculated for each SNP by counting the number of times the SNP was detected in a $\mathrm{ROH}$ across the dataset of the whole population.

\section{Linkage disequilibrium and haplotype blocks}

As a measurement of LD, we used the two most commonly used statistics, $D^{\prime}$ and $r^{2}$, for an easy comparison of our results with those of other reports. Haploview v4.2 [29] was used to estimate LD. For each breed, each chromosome was analysed and all pairwise LD combinations $\left(D^{\prime}\right.$ and $\left.r^{2}\right)$ were estimated. SNP pairs were grouped according to their pairwise distance into 14 categories: <10 kb, $10-20 \mathrm{~kb}, 20-40 \mathrm{~kb}, 40-60 \mathrm{~kb}, 60-100 \mathrm{~kb}$, $100-200 \mathrm{~kb}, 200-500 \mathrm{~kb}, 500 \mathrm{~kb}-1 \mathrm{Mb}, 1-2 \mathrm{Mb}, 2-5 \mathrm{Mb}$, 5-10 Mb, 10-20 Mb, 20-50 Mb, and >50 Mb. Average LD within each group was calculated for each breed. Average LD with neighbouring pairs of SNPs and average LD across the chromosome were also estimated for each chromosome. For non-syntenic SNPs, a subset of SNPs from the whole genome was used to estimate LD. For each autosome, a random representative sample of SNPs was selected to obtain an estimate of LD ( $5 \%$ of the SNPs for each autosome). Haploview v4.2 was also used to identify the haplotype blocks present in each chromosome. Haplotype blocks were defined using the method described in [30]. Two SNPs were considered to be in 
strong LD if the upper one-sided $95 \%$ confidence bound of $D^{\prime}$ was higher than 0.98 and if the lower bound was higher than 0.7 .

\section{Effective population size and inbreeding coefficients} Effective population size $\left(N_{e}\right)$ was calculated for each breed using the default parameters and the random mating model in the software NEESTIMATOR v2 [31]. A bias-corrected version of the LD method [32] was used to obtain the final estimate of $N_{e}$.

Marker-based inbreeding coefficients for each breed were estimated using the GCTA software [33]. Individual allele frequencies for each population were calculated and three different metrics for $F$ values were calculated by GCTA: $F_{1}$ based on the variance of the additive genotype; $F_{2}$ based on the excess of homozygotes; and $F_{3}$ based on the correlation between uniting gametes [33]. Inbreeding coefficients for each breed were calculated by averaging inbreeding coefficients of all individuals for that breed.

\section{Results}

\section{Descriptive statistics}

From the initial set of 54,241 SNPs, 1450 (2.69 \%) nonautosomal SNPs and 314 SNPs that had no chromosome assignment were removed. Another 3837 SNPs were excluded: 1662 because minor allele frequency (MAF) was $<0.01,1838$ because SNP call rate $\left(\mathrm{CR}_{\mathrm{SNP}}\right)$ was $<0.95$, and 337 because they deviated from the HWE. Fortyone SNPs could not be mapped to the ovine Genome Assembly v3.1 and were removed from the analysis. No individual was removed due to low call rates $\left(\mathrm{CR}_{\mathrm{IND}}\right)$. A total of 48,599 SNPs across the five populations met the filtering criteria and were included in the final analysis. The distributions of the SNPs after filtering and the average distances between adjacent SNPs on each chromosome are in Additional file 1: Table S1. SNPs were uniformly distributed across all autosomes since marker density was similar for all chromosomes with the average distance between SNPs ranging from $47.26 \mathrm{~kb}$ on OAR8 (OAR for Ovis aries chromosome) to $61.62 \mathrm{~kb}$ on OAR24. Autosomes varied in size, with OAR24 being the shortest $(42.03 \mathrm{Mb})$ and OAR1 the longest chromosome (275.61 Mb). After filtering, the number of SNPs on each chromosome ranged from 683 on OAR24 to 5484 on OAR1. The distribution of MAF differed between populations (see Additional file 1: Table S1; Additional file 2: Figure S1). Additional file 2: Figure S1 shows that BL and PD had an excess of SNPs with a low MAF $(<0.1)$ compared to MER and the two crossbred populations.

\section{Genetic diversity and population structure}

Analysis of genetic diversity using the average observed heterozygosity and average expected heterozygosity (Table 1) showed that genetic diversity was lowest for BL, with $H_{e}$ and $H_{o}$ estimates both equal to 0.30 , followed by PD, with $H_{e}$ and $H_{o}$ estimates both equal to 0.34. Among the pure breeds, MER was the most diverse with $H_{e}$ and $H_{o}$ estimates both equal to 0.38 . Compared to the pure breeds, the crosses had a higher level of genetic diversity. We also investigated the level of relatedness between populations by calculating the pairwise $F_{\mathrm{ST}}$ (Table 1). The two most distantly related breeds are BL and MER with an average pairwise $F_{\mathrm{ST}}$ value of 0.062 . The smallest average pairwise $F_{\mathrm{ST}}$ was observed between the two crosses $\left(F_{\mathrm{ST}}=0.013\right)$, which were the most closely related pair. These estimates reflected expectations since MER and $\mathrm{BL}$ contributed to both crosses. Higher average $F_{\mathrm{ST}}$ values were observed for every pair-wise comparison of populations involving MER. Figure 1 shows the relationship between the first two principal components, which explained $91.7 \%$ of the total variation and separated the populations into their respective breed groups.

\section{Runs of homozygosity}

The number of ROH differed significantly between populations. BL had the largest total number of $\mathrm{ROH}(12,561)$ followed by PD (9875) and MER (2008). There were

Table 1 Genetic diversity within the five sheep populations studied

\begin{tabular}{|c|c|c|c|c|c|c|c|}
\hline & $F_{\mathrm{ST}}$ & & & & $\%$ of polymorphic SNPs & $H_{e}$ & $H_{o}$ \\
\hline & PD & MER & BxM & $B \times M x P$ & & & \\
\hline$B L$ & 0.053 & 0.062 & 0.019 & 0.033 & 99.21 & 0.30 & 0.30 \\
\hline PD & & 0.053 & 0.036 & 0.016 & 98.74 & 0.34 & 0.34 \\
\hline MER & & & 0.045 & 0.043 & 99.86 & 0.38 & 0.38 \\
\hline BxM & & & & 0.013 & 99.89 & 0.37 & 0.403 \\
\hline$B \times M \times P$ & & & & & 99.96 & 0.38 & 0.404 \\
\hline
\end{tabular}

$H_{e}$ expected heterozygosity

$H_{o}$ observed heterozygosity

For each SNP and for each breed, $H_{e^{\prime}} H o$, and $F_{S T}$ were estimated and averaged 


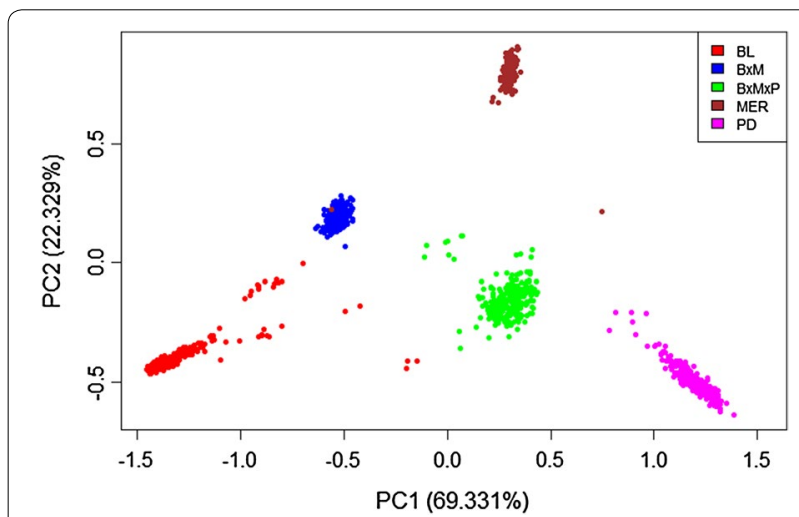

Fig. 1 PCA plot of the genetic relationship matrix for the five populations studied. The three pure breeds were clearly separated and the first two principal components explained about $91.7 \%$ of the total variation

49.65, 37.89 and 7.57 ROH per animal for the BL, PD and MER breeds, respectively. As expected, we found that the pure breeds had more $\mathrm{ROH}$ across the whole genome than the crosses. In fact, $\mathrm{MxB}$ and MxBxP had, in total, only 80 and $331 \mathrm{ROH}$. Figure 2 shows the sum of $\mathrm{ROH}$ (in $\mathrm{Mb}$ ) per animal genome for each of the five populations. There were clear differences in both the levels and variation of $\mathrm{ROH}$ frequency. BL and PD had a larger sum of $\mathrm{ROH}$ per animal genome than MER and the crossbred populations.

Among the 1273 animals, 1015 (80 \%) had at least one $\mathrm{ROH}$ longer than $1 \mathrm{Mb}$ and 751 (59\%) had at least one $\mathrm{ROH}$ longer than $5 \mathrm{Mb}$. If we consider only the pure breeds, then all animals had at least one $\mathrm{ROH}$ longer than $1 \mathrm{Mb}$ and 688 of the 782 animals (88 \%) had at least one $\mathrm{ROH}$ longer than $5 \mathrm{Mb}$. There are clear differences between breeds in the frequencies of the $\mathrm{ROH}$ of various lengths (Fig. 3). Two breeds, BL and PD, had on average, a larger part of their genome that contained $\mathrm{ROH}$ of 1 to $5 \mathrm{Mb}(\mathrm{BL}=126.06 \mathrm{Mb}$ and $\mathrm{PD}=94.88 \mathrm{Mb})$. In all five populations, most of the $\mathrm{ROH}$ were shorter and ranged from 1 to $10 \mathrm{Mb}$ (Fig. 3). No crossbred animal had a ROH longer than $20 \mathrm{Mb}$. In our study, the three animals with the highest level of homozygosity were PD individuals with $427.2,410.5$ and $396.45 \mathrm{Mb}$ of their genome classified as ROH (data not shown), which is close to $20 \%$ of the genome.

OAR2 had the largest number of ROH (3381 for 543 animals) with on average $11.89 \%$ of the chromosome consisting of $\mathrm{ROH}$. Generally, the number of $\mathrm{ROH}$ per chromosome tended to decrease with chromosome length (Fig. 4). The largest proportion of the genome in $\mathrm{ROH}$ was observed for OAR25 and OAR22 with 16.48 and $15.05 \%$, respectively. SNP OAR2_119604666.1 on OAR2 was the most frequently found in $\mathrm{ROH}(174$ occurrences); followed by OAR10_26604546.1 and s35658.1 (both on OAR10) with 142 and 128 occurrences. We also investigated LD between SNPs that were located in the vicinity of these three SNPs using Haploview [29] and found that most SNPs in these regions were in high LD with each other. In addition, OAR4, 6 and 15 contained SNPs that occurred at high frequencies in $\mathrm{ROH}$ (Fig. 5). In this data, no $\mathrm{ROH}$ were found on OAR24 and 26.

\section{Linkage disequilibrium analysis}

Estimates of LD based on $r^{2}$ values were different for each of the five populations (Fig. 6). Short-range LD was observed in all populations but the rate of LD decay differed between populations. LD was present over larger distances in the BL and PD populations but dropped quickly with increasing distance in the MER and the two crossbred populations. For SNPs up to $10 \mathrm{~kb}$ apart, the average $r^{2}$ values were equal to 0.34 (BL), 0.27 (MER), $0.33(\mathrm{PD}), 0.29(\mathrm{MxB})$, and $0.3(\mathrm{MxBxP})$. Average LD between adjacent SNPs per chromosome was also estimated and some variation in the extent of LD on different chromosomes was observed for the five populations. In each population, the maximum average LD between adjacent SNPs was observed on OAR10 and was equal to 0.25 (BL), 0.15 (MER), $0.21(\mathrm{PD}), 0.17(\mathrm{MxB})$, and 0.16 $(\mathrm{MxBxP})$. However, the minimum average LD between adjacent SNPs was found for different chromosomes in the five populations (see Additional file 1: Table S2).

Linkage disequilibrium for each population was also estimated for all possible pairs of SNPs on the autosomes using $D^{\prime}$. Similarly to the $r^{2}$ values, $D^{\prime}$ also decreased as pairwise marker distances increased; however, the rate of decay was less pronounced for $D^{\prime}$ than for $r^{2}$ (see Additional file 2: Figure S2). More details about LD analysis using $\mathrm{D}^{\prime}$ are included in Additional file 1: Tables S3 and S4 and Additional file 2: Figure S2.

Average $r^{2}$ and $D^{\prime}$ values for all possible pairs of SNPs on each chromosome were calculated and an inverse relationship between chromosome length and average $D^{\prime}$ was observed for each population (see Additional file 1: Tables S4, S5).

\section{Pattern of haplotype blocks by breed}

Haplotype blocks were identified using Haploview v4.2 [29] following the methods described in [30]. Table 2 summarises the distribution of haplotype blocks in the five populations. The total number of haplotype blocks ranged from 1581 (for $\mathrm{MxB}$ ) to 2534 (for PD). Although the PD genome had the largest number of haplotype blocks, the percentage of the genome covered by haplotypes was greater for BL than for PD (177.74 Mb and 7.25 $\%$ for $\mathrm{BL}$ and $130.53 \mathrm{Mb}$ and $5.32 \%$ for $\mathrm{PD})$. The longest 


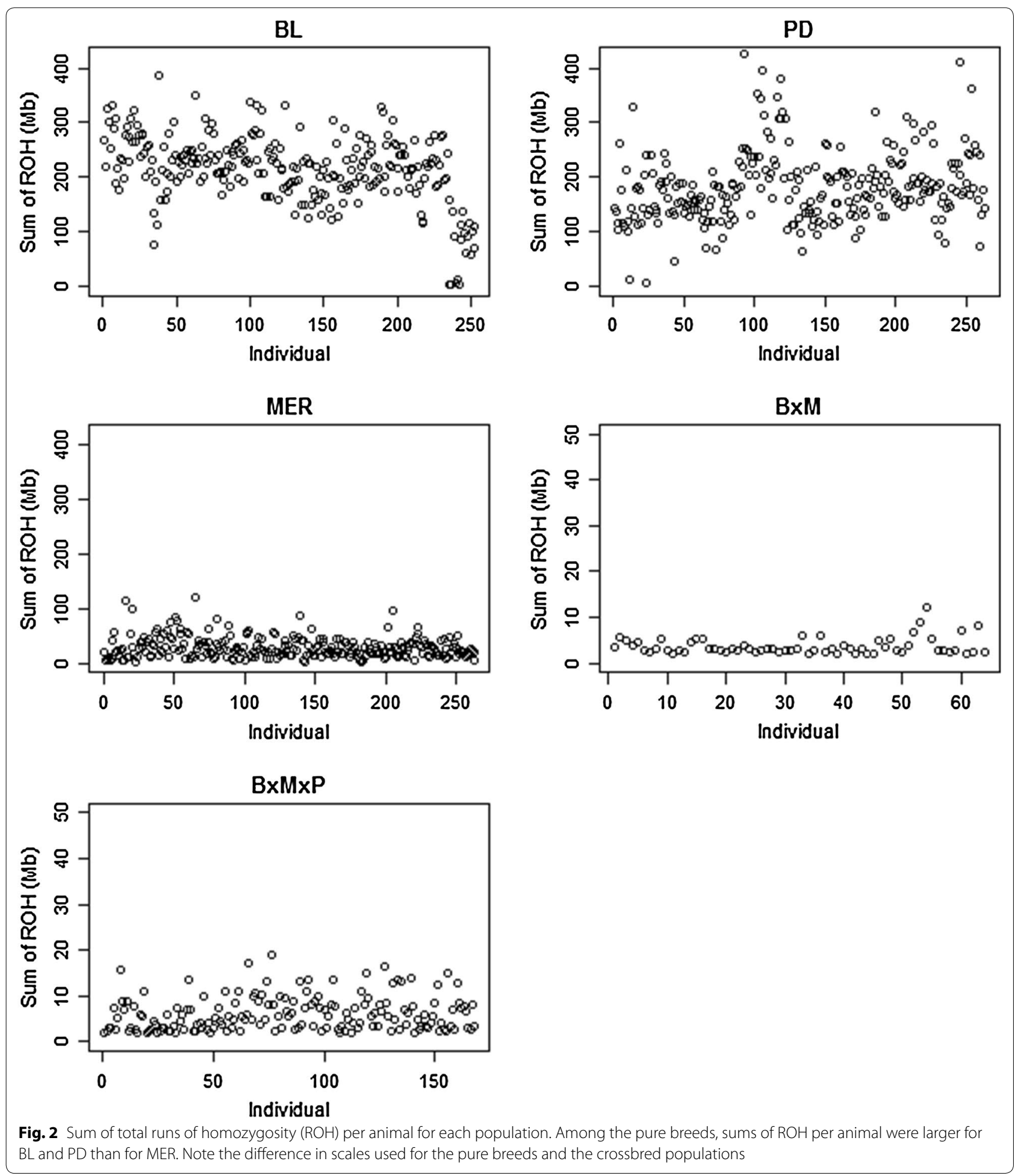

block was detected on OAR10 with a length of nearly $500 \mathrm{~kb}$ for all populations except for BL for which the longest haplotype block was on OAR21 ( 500 kb). The shortest haplotype blocks were on OAR12 for $\mathrm{BL}, \mathrm{MxB}$ and MxBxP (1.95 kb), OAR5 for MER (0.06 kb) and OAR26 for PD (1.04), but these mostly just reflected the few SNP pairs that were at close distances on the array. The number of SNPs within a block varied between 2 


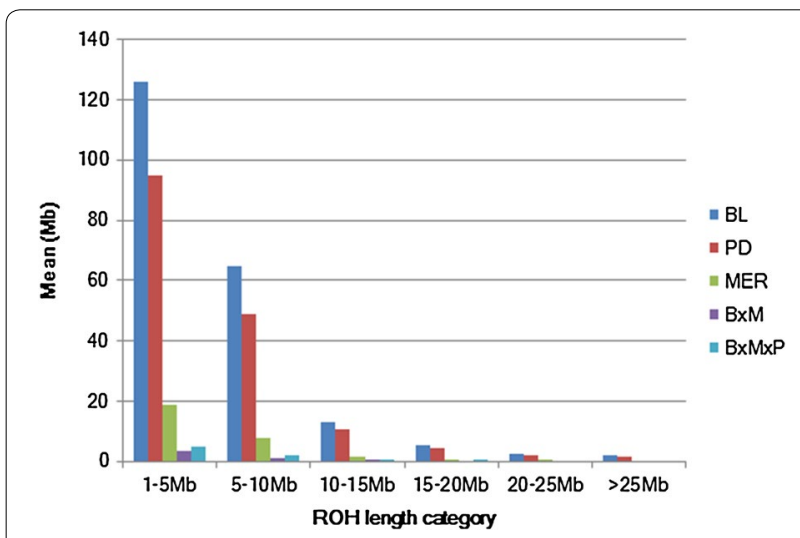

Fig. 3 Average $\mathrm{ROH}$ per population. The average length of $\mathrm{ROH}$ (in $\mathrm{Mb}$ ) for each population across six interval sizes. For each animal, within each $\mathrm{ROH}$ length category, $\mathrm{ROH}$ were summed up and then averaged per population

and 12 whereas the percentage of SNPs in blocks ranged from 7.10 (for $\mathrm{MxB}$ ) to 15.13 \% (for $\mathrm{BL}$ ). Chromosomewise percentage coverage of blocks is in Fig. 7 and for more details (see Additional file 1: Table S6). For all populations except BL, OAR10 had the highest percentage of coverage by haplotype blocks. BL also had a large number of haplotype blocks on OAR10 but this was even larger on OAR7.

\section{Effective population size and inbreeding coefficients}

The size of $N_{e}$ differed between populations. BL had the smallest estimated $N_{e}$ whereas MER was much more diverse (Table 3). As expected, the two crossbred populations were also quite diverse.

The average inbreeding estimates ( $F$ values) were calculated based on the SNP genotypes for all five populations (Table 3). No significant inbreeding was observed in the pure breeds and some outbreeding was observed in the crossbred populations. Estimated $F_{2}$ values were based on the excess of homozygotes for BL, MER and PD ranged from -1.29 to 0.22 , from -0.19 to 0.13 , and from -1.25 to 0.23 with median values $0.09,-0.013$ and 0.02 , respectively, for details (see Additional file 1: Table S7).

\section{Discussion}

In this study, we identified clear but modest genetic differences between three pure sheep breeds MER, BL

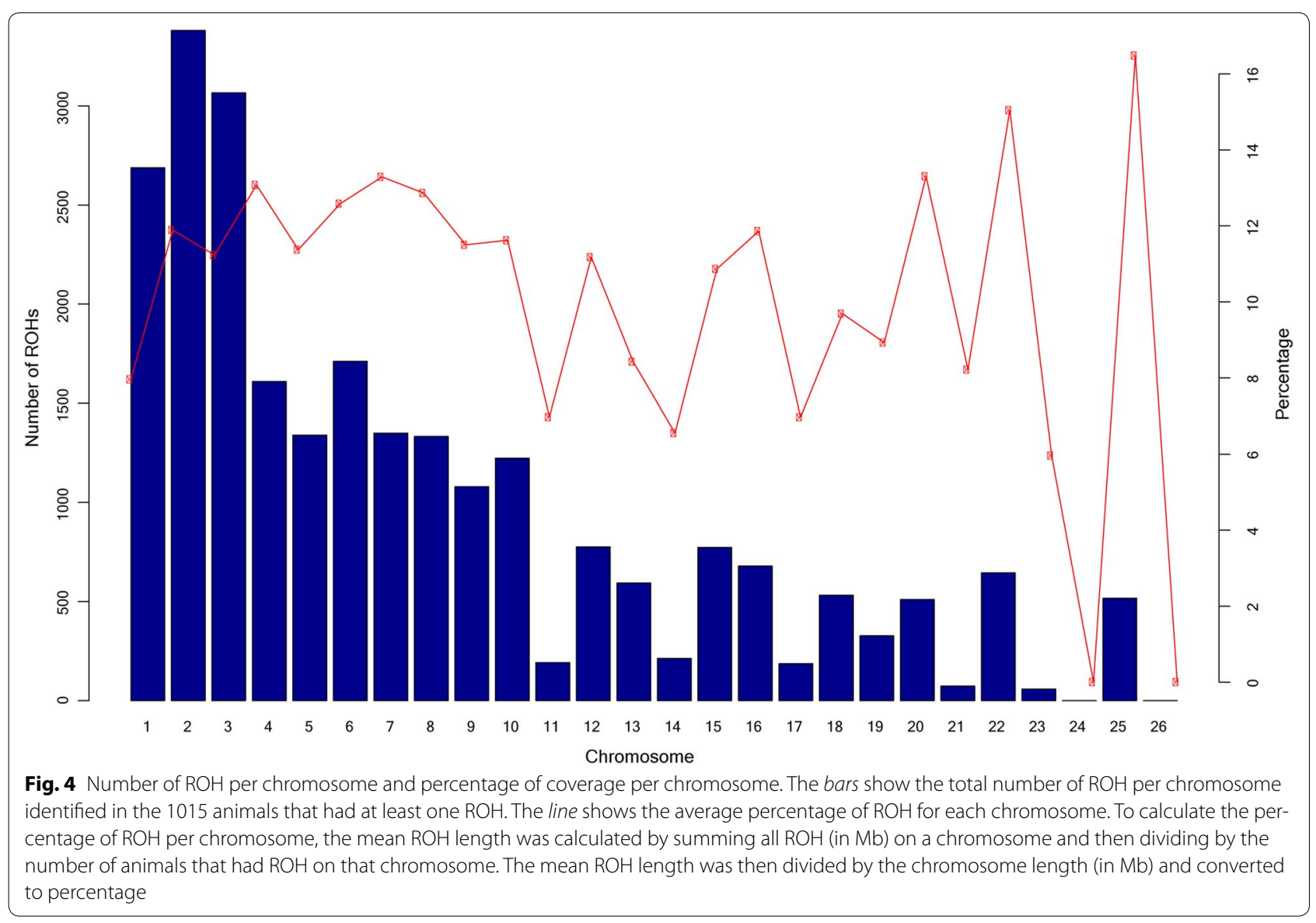




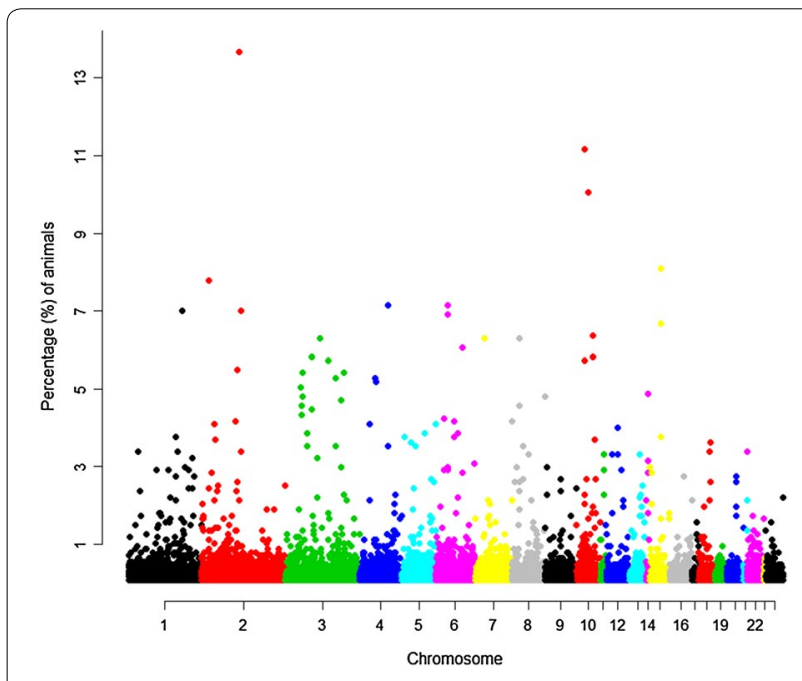

Fig. 5 Incidence of each SNP in a $\mathrm{ROH}$. Note that the last chromosome included in this figure is OAR25 because no $\mathrm{ROH}$ were identified on OAR24 and 26

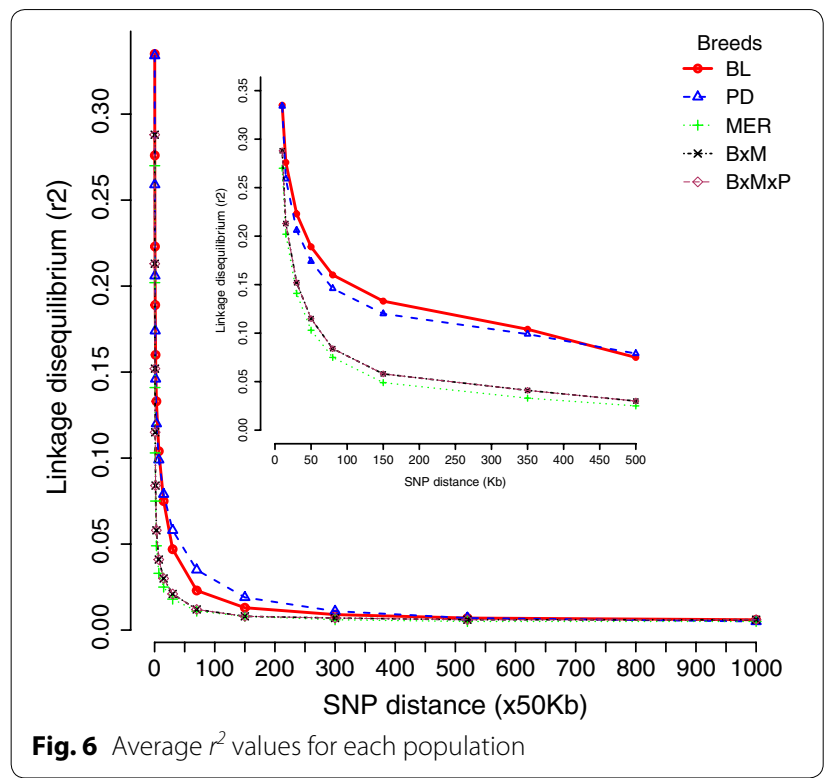

and PD. Analysis of genetic diversity within these three populations (Table 1) showed that MER had the highest level of diversity with an estimated gene diversity $\left(H_{\mathrm{e}}\right)$ of 0.38 and $99.86 \%$ polymorphic SNPs. This fits well with the known history of the breed. The foundation of the Australian Merino population involves contributions from different European, Asian and African breeds [22] and, therefore, Australian Merino are a combination of strains of sheep rather than an ancient single homogenous breed. Furthermore, Australian Merinos were reported as the most diverse sheep population [22]. The higher rate of LD decay in MER confirms its high level of genetic diversity. In contrast to MER, BL and PD had lower levels of genetic diversity and a lower rate of LD decay. BL was developed in England during the 17th century from a founding stock of Dishley Leicester rams that were imported into Australia in 1871 [34]. PD sheep were developed in Australia from the Dorset sheep breed between 1937 and 1954 with the aim of breeding Dorset sheep without horns. In both cases, the populations underwent bottlenecks during breed formation which accounts for their smaller $N_{e}$ sizes [34].

The extent and pattern of genome-wide LD are important for QTL mapping of production traits in association studies, i.e., it is the strength of LD between markers and QTL that provide the statistical power to detect associations in GWAS. In our analysis, BL and PD had a significantly higher average LD compared to MER with the latter also showing a higher rate of LD decay. Figure 6, Additional file 2: Figure S2 and Additional file 1: Table S8 clearly illustrate the differences of the two meat breeds ( $\mathrm{PD}$ and $\mathrm{BL}$ ), which had a higher average LD with the wool breed (MER), which had the lowest average LD. As expected the crosses $(\mathrm{MxB}$ and $\mathrm{MxBxP})$ had an intermediate average LD compared to the meat and wool breeds. Our results indicate that LD decay was breed-specific, which is in agreement with other reports in sheep [22] and other species $[35,36]$. The high LD in BL and PD is most likely attributable to the smaller $N_{e}$ size of $\mathrm{BL}$ $\left(N_{e}=140\right)$ and $\mathrm{PD}\left(N_{e}=152\right)$ in comparison to MER $\left(N_{e}=348\right)$. The $N_{e}$ sizes of BL and PD are similar to those reported for Spanish Churra sheep $\left(N_{e}=128\right)$ [23] and for Finnsheep ( $N_{e}=119$ and 122) [37]. However, the $N_{e}$ reported in our study are significantly smaller than those reported by the SheepHapMap project [1] $(\mathrm{MER}=853$, $\mathrm{PD}=318, \mathrm{BL}=242)$ which were on average 2.1 times larger than our figures; but most of the sheep breeds analysed in the SheepHapMap project had large $N_{e}$ [1], i.e., 600 for Spanish Churra and 795 for Finnsheep breeds. This may be due to the fact that the SheepHapMap project used fewer and unrelated animals, whereas in our study some half-sib animals were used and the average number of animals/sire ranged from 1.61 (for PD) to 2.38 (for MER). However, the rankings of $N_{e}$ agree, with the largest and smallest $N_{e}$ for MER and BL, respectively.

Comparison between $r^{2}$ and $D^{\prime}$ values revealed that at the same genomic distance, the average $D^{\prime}$ value was larger than the average $r^{2}$ value, which is in agreement with previous reports [22, 23]. This might be attributed to the presence of rare alleles and unobserved haplotypes that could inflate $D^{\prime}$ but not $r^{2}$ [38]. Typically, $r^{2}$ is the preferred measure of LD in the context of QTL mapping, whereas $D^{\prime}$ is the measure of choice to assess recombination patterns. Taken together, both $D^{\prime}$ and $r^{2}$ showed 


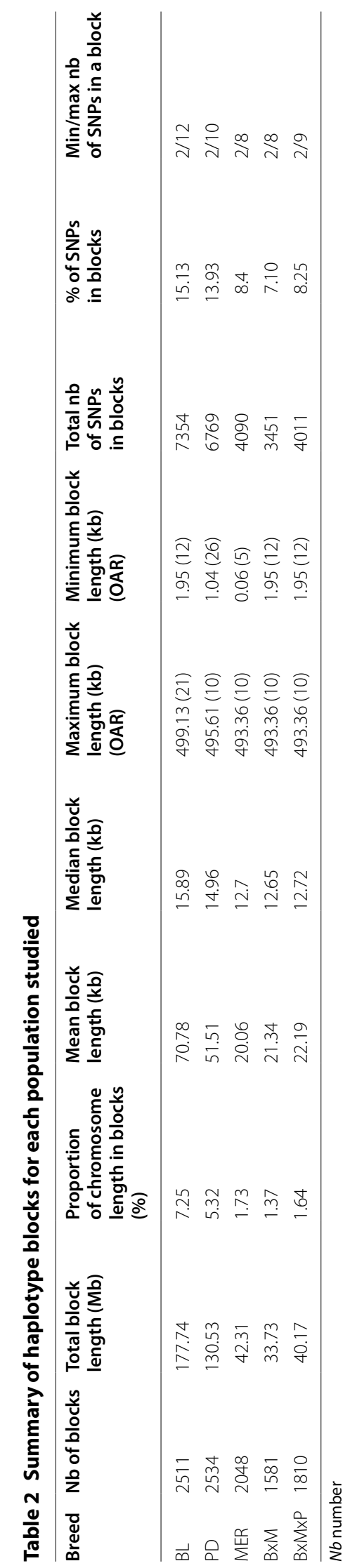




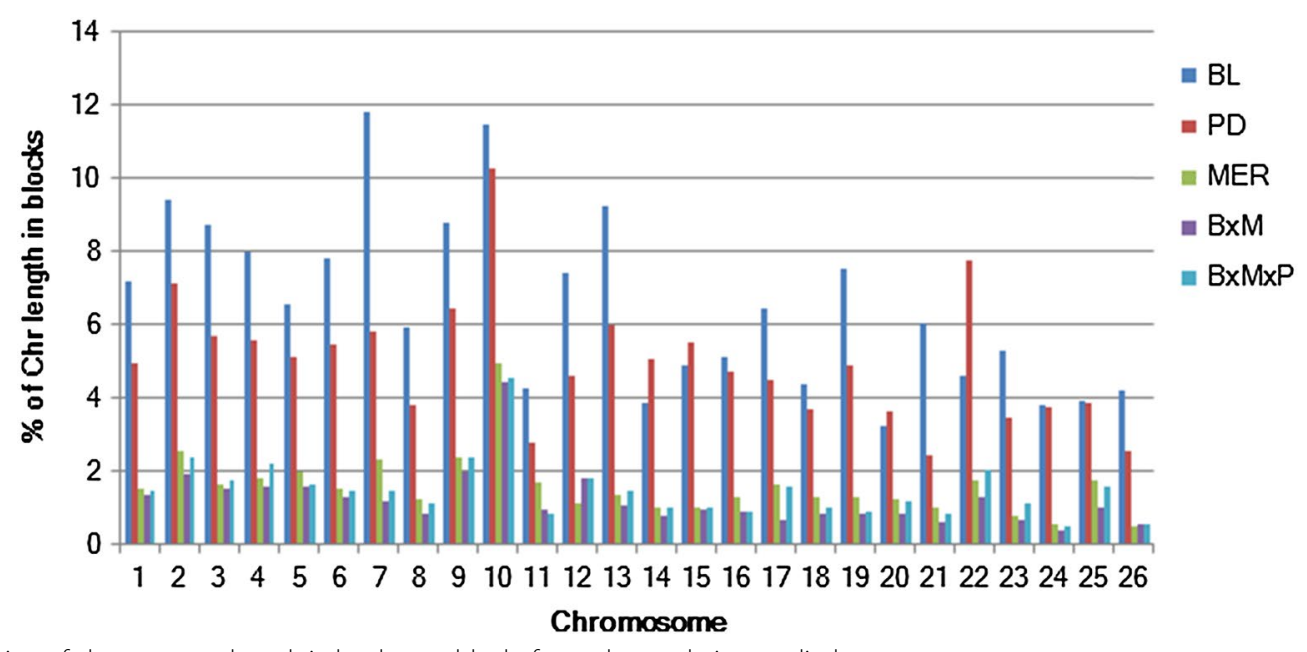

Fig. 7 Proportion of chromosome length in haplotype blocks for each population studied

Table 3 Average inbreeding coefficient and current effective population size for each population studied

\begin{tabular}{|c|c|c|c|c|c|c|c|}
\hline \multirow[t]{2}{*}{ Breed } & \multirow[t]{2}{*}{$N_{e}$} & \multicolumn{3}{|c|}{ Inbreeding coefficient } & \multicolumn{3}{|c|}{ Critical percentage } \\
\hline & & $F_{1}$ & $F_{2}$ & $F_{3}$ & $F_{1}$ & $F_{2}$ & $F_{3}$ \\
\hline BL & 140 & -0.006 & 0.002 & 0.002 & 16.996 & 58.498 & 1.186 \\
\hline PD & 152 & -0.013 & -0.001 & -0.001 & 15.909 & 28.409 & 4.167 \\
\hline MER & 348 & -0.001 & 0.0004 & 0.0004 & 1.509 & 0 & 0 \\
\hline $\mathrm{M} \times \mathrm{B}$ & 272 & -0.085 & -0.084 & -0.084 & 0 & 0 & 0 \\
\hline MxBxP & 152 & -0.057 & -0.057 & -0.057 & 2.597 & 0 & 0 \\
\hline
\end{tabular}

Inbreeding coefficient was calculated for each individual and then averaged across the populations

$F_{1}$ is calculated based on the variance of the additive genotype

$F_{2}$ is calculated based on the excess of homozygotes

$F_{3}$ is calculated based on the correlation between uniting gametes

Critical percentage is the percentage of individuals having an $F$ value $>0.065$

that beyond $20 \mathrm{Mb}$ the average LD values for all populations are very similar to the average value of non-syntenic SNPs (see Additional file 1: Table S8), i.e., there was no LD. Previous reports on sheep with microsatellite markers reported that LD is very high and extends over a considerable range up to $20 \mathrm{cM}$ and that high LD exists between marker pairs that are separated by $<5 \mathrm{cM}[22$, 39]. However, a recent study by Kijas et al. [21] on sheep using SNP arrays reported that LD decays faster and at much shorter genomic distances. This is in line with what we observed here. We found that beyond $0.5 \mathrm{Mb}$, the average $r^{2}$ and $D^{\prime}$ values dropped to $<0.01$ and 0.45 , respectively, for most breeds; the useful LD [40] did not extend beyond $0.5 \mathrm{Mb}$. Very similar results were found in a bovine whole-genome LD analysis [15]. In sheep, a recent assessment of LD in Spanish Chura also reported comparable levels of LD with an average $r^{2}$ value of 0.329 for SNPs that are up to $10 \mathrm{~kb}$ apart and an average $r^{2}$ value of 0.061 for SNPs that are separated by $200-500 \mathrm{~kb}$
[23]. Kijas et al. [21] reported LD over short distances using the $700 \mathrm{k}$ sheep SNP array and showed that $r^{2}$ values at $10 \mathrm{~kb}$ were equal to $0.186,0.279$ and 0.339 , respectively, for MER, PD and BL, while we obtained $r^{2}$ values of 027, 0.334 and 0.335 . Both studies were in close agreement for BL but we obtained higher estimates of LD for MER and PD, which is due to the $50 \mathrm{k}$ array including too few SNPs that are $10 \mathrm{~kb}$ apart to reliably estimate $\mathrm{LD}$ at short distances.

Comparison of our results with those of other studies on LD in other species shows that LD in sheep persists for relatively shorter genomic distances than in cattle, pigs or dogs. While LD estimates are not immediately comparable because the number of samples, the number of markers and the types of measurements vary between reports, the emerging picture is that sheep have lower LD than other domestic animals. The extent of LD in various cattle breeds is greater than that found in this study $[16,17,41,42]$. LD analysis in six commercial pig lines 
showed that the average $r^{2}$ values only dropped to $<0.01$ when the interval between SNPs was greater than $3 \mathrm{cM}$ [43]; another study on four pig breeds, reported a rather high $r^{2}$, i.e., between 0.19 and 0.26 for SNPs separated by $0.5 \mathrm{Mb}$ [44]. Similarly, LD extends up to several Mb for dog breeds [45]. In contrast, LD in human populations extends to only tens of kb [46]. This agrees with Kijas et al. [1] who indicated that sheep have been less intensively selected than other domestic species and sampled from a larger initial gene pool. In Australia, in comparison to other livestock industries, selection pressure on sheep populations has not been as strong as for e.g. dairy cattle, which accounts for the lower levels of LD and larger $N_{e}$ observed in this study.

Chromosome-wise mean LD varied between breeds and between chromosomes (see Additional file 1: Tables S4, S5), which could be due to differences in recombination rates, heterozygosity, genetic drift and effects of selection between chromosomes and breeds [47]. Chromosome-wise average $\mathrm{LD}$ values were larger for $\mathrm{BL}$ and PD than for MER and the crossbred populations. Chromosome-wise average LD values were in agreement with the haplotype block structure and the $\mathrm{ROH}$ distribution across the sheep genome (Figs. 4, 8). Chromosomes that showed higher average $r^{2}$ values also had a larger proportion of haplotype blocks and $\mathrm{ROH}$. This was especially the case for OAR10, 22 and 23.

Higher levels of homozygosity and LD are expected in genomic regions that have undergone intensive selection for a particular trait (a signature of selection, as in, e.g., $[1,48])$. It is also expected that these regions will have longer haplotype blocks. In the populations studied here, OAR10 was the chromosome with the largest proportion in haplotype blocks. SNP OAR10_26604546.1, which had the second largest number of occurrences in $\mathrm{ROH}$ counts, is on this chromosome. A total of $142(11.15$ $\%$ ) animals had at least one ROH near this SNP in the region between 26.7 and $29.3 \mathrm{Mb}$. This region contains the RXFP2 (relaxin/insulin-like family peptide receptor 2) gene, which is associated with the absence of horns in sheep [49] and for which there is also strong evidence of selection in cattle [50]. A QTL associated with horns (HO) in Soay sheep peaked at $27.1 \mathrm{cM}$ on OAR10 [51, 52]. We found that BL and PD had very long haplotype blocks in this region, but MER (and the crossbred populations) had shorter blocks. PD had three long haplotype blocks of 495.6, 387 and $195 \mathrm{~kb}$ in the region between 28.5 and $31.1 \mathrm{Mb}$ on OAR10 that contained nine, ten and six SNPs, respectively. In the same region between 28.4 and 30.4 Mb on OAR10, BL had four haplotype blocks that were 123.5, 182.8, 479 and $209.7 \mathrm{~kb}$ long and each contained six SNPs. In contrast, MER had four small blocks (18.2, 114.8, 8.7 and $10.5 \mathrm{~kb})$ with two, four, two and two SNPs, respectively. For completeness, MxB had one block of $90 \mathrm{~kb}$ that contained four SNPs and MxBxP had two blocks (18.2 and $151.29 \mathrm{~kb}$ ) that contained two and five SNPs, respectively. The significant difference in haplotype block length suggests that selection on this region has been intensive in the $\mathrm{PD}$ and $\mathrm{BL}$ breeds.

OAR2 had the largest number of $\mathrm{ROH}$ and a large proportion of genome within haplotype blocks. This chromosome contains SNP OAR2_119604666.1 which had the largest number of occurrences in $\mathrm{ROH}$ (Fig. 4). Near this SNP, $174(13.67 \%)$ animals had at least one $\mathrm{ROH}$ that spanned the region between 109.1 and $111.3 \mathrm{Mb}$. The HERC2-like gene i.e. LOC101102534 (between 112.4 and $112.7 \mathrm{Mb}$ ) is located near this region and has been reported to affect hair colour and skin pigmentation in humans [53]. Our sheep populations were white wool sheep breeds and this region on OAR2 could be under selection.

OAR15 also has a region between 50.21 and $53.04 \mathrm{Mb}$ that contains SNPs that had large numbers of occurrences in the $\mathrm{ROH}$ counts. A total of 109 (8.56\%) animals had at least one $\mathrm{ROH}$ that spanned this region. Among these 109 animals, 103 contained SNP s07957.1 (at 50 218 062) in their ROH segments. In this region, PD had a long haplotype block $(399.99 \mathrm{~kb})$ of 5 SNPs and BL has two long haplotype blocks of 280.63 and $413.29 \mathrm{~kb}$ of six SNPs each. In contrast, MER had two small haplotype blocks of 16.35 and $9.5 \mathrm{~kb}$ of two SNPs each. We explored a $1 \mathrm{Mb}$ region (49.2 to $51.2 \mathrm{Mb}$ ) in each direction from the SNP s07957.1 on OAR15 and found that this region contains 39 protein coding genes, which represents about $3.6 \%$ of the total number of genes present on OAR15 (1098 genes). The list of the genes with their description and summary functions of their human homologs is in Additional file 1: Table S9.

Genetic diversity and therefore genome-wide LD information is important for genomic selection studies. Genomic selection (GS) uses genetic markers that cover the whole genome to predict genomic estimated breeding values (GEBV). In genomic selection, the accuracy of GEBV depends largely on the heritability of the trait, its genetic architecture and the effective size of the targeted populations. The low levels of LD and high levels of diversity observed in these populations suggest that accuracies of prediction in sheep could be lower than in other populations that have higher LD under similar trait/ population scenarios. As proposed by Kijas et al. [21], denser marker panels may help offset the effects of low LD. Larger datasets with more animals than the numbers used in, e.g., cattle or pig may also be required to obtain similar levels of prediction accuracy as those observed in these other species. This may be particularly true for the Merino population. 


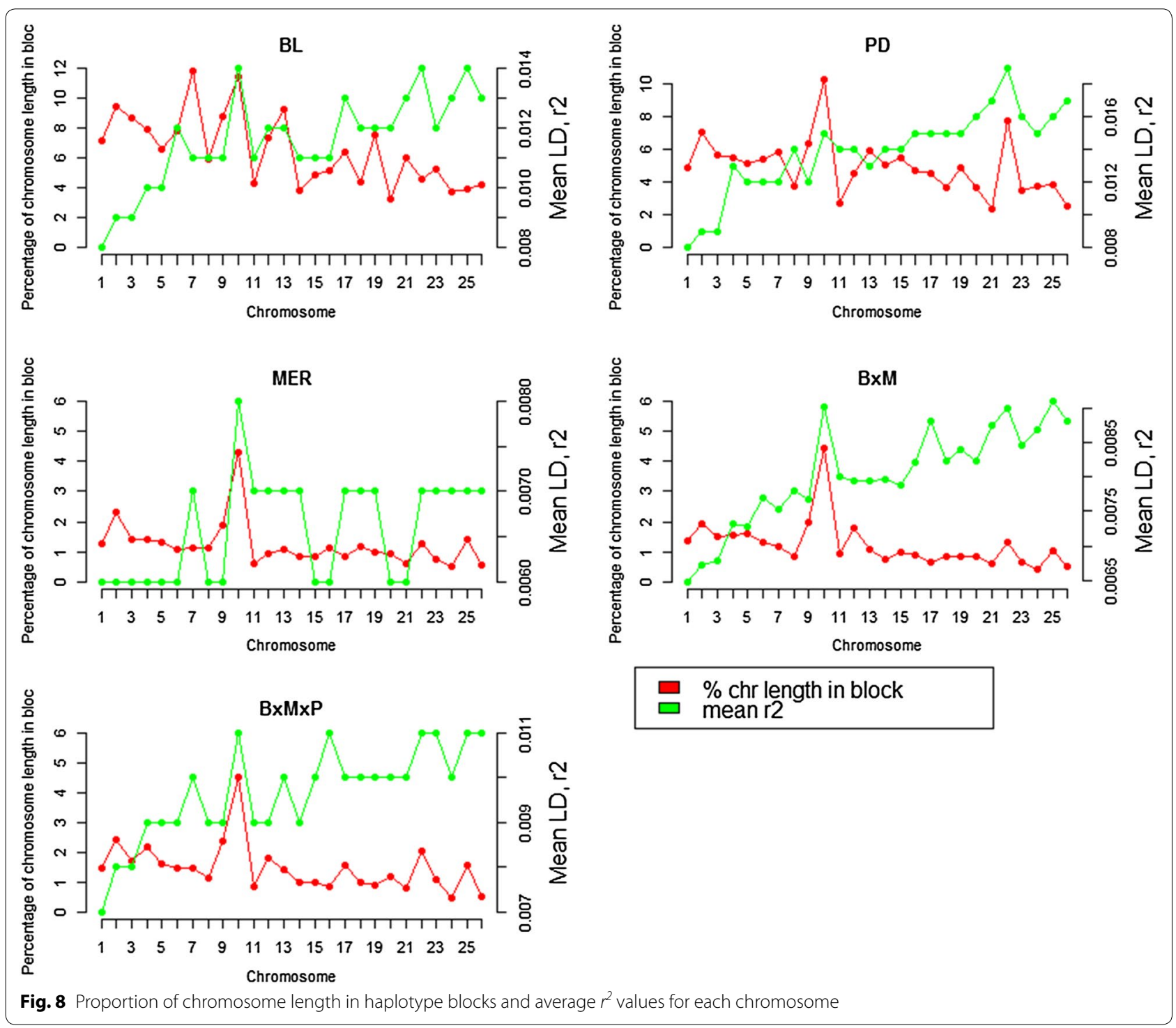

\section{Conclusions}

We estimated and compared linkage disequilibrium (LD) and several other genetic diversity parameters, including gene diversity $\left(H_{e}\right)$ and fixation index $\left(F_{\mathrm{ST}}\right)$, in five Australian sheep populations i.e. the three most economically important pure breeds and two crosses of these pure breeds. LD decayed rapidly in all populations but the rate of decay varied significantly between them. While genetic distances between breeds were relatively modest in comparison to other livestock species, the genetic diversity within Merino was high. The results of this study improve our understanding of the genetic diversity in the three main Australian sheep breeds and will be useful to perform effective GWAS studies. Our results also provide insights into the influence of selection within these breeds and provide useful knowledge that will contribute to design appropriate and successful genomic selection programs.

\section{Additional files}

Additional file 1: Table S1. Summary statistics for the SNPS, average minor allele frequency and heterozygosity. Table S2. Average linkage disequilibrium ( $r 2$ ) between adjacent markers on the autosomes (OAR). Table S3. Average linkage disequilibrium ( $\left.D^{\prime}\right)$ between adjacent markers on the autosomes (OAR). Table S4. Chromosome-wise average linkage disequilibrium ( $D^{\prime}$ ) for each population studied. Table S5. Chromosomewise average linkage disequilibrium ( $r 2$ ) for each population studied.

Table S6. Summary of the chromosome-wise haplotype analysis. Table S7. Range of inbreeding coefficients for each population studied. Table S8. Mean linkage disequilibrium in the five populations at varying map distances. Table S9. List of the genes located in the region between 49.2 and $51.2 \mathrm{Mb}$ on OAR15.

Additional file 2: Figure S1. Distribution of minor allele frequency (MAF) for each population studied. The percentage of SNP is plotted for each frequency bin. Figure S2. Average D'values for each population. 


\section{Authors' contributions}

HAM and CG designed the project and carried out the analyses, CG, SC and PK advised on the project. All authors read and approved the final manuscript.

\section{Author details}

${ }^{1}$ School of Environmental and Rural Science, University of New England, Armidale, NSW 2351, Australia. ${ }^{2}$ School of Science and Technology, University of New England, Armidale, NSW 2351, Australia.

\section{Acknowledgements}

This project was funded by the Sheep Cooperative Research Centre (Sheep (RC), Australia. CG and PK were supported by an Australian Research Council Discovery Project DP130100542. CG and HAM also acknowledge support by a Grant from the Next-Generation BioGreen 21 Program (No. PJ01134906), Rural Development Administration, Republic of Korea.

\section{Competing interests}

The authors declare that they have no competing interests.

Received: 16 December 2014 Accepted: 2 November 2015

Published online: 24 November 2015

\section{References}

1. Kijas JW, Lenstra JA, Hayes B, Boitard S, Porto-Neto LR, San Cristobal $M$, et al. Genome-wide analysis of the world's sheep breeds reveals high levels of historic mixture and strong recent selection. PLoS Biol. 2012;10:e1001258.

2. Arranz JJ, Bayon Y, Primitivo FS. Genetic variation at microsatellite loci in Spanish sheep. Small Rumin Res. 2001;39:3-10.

3. Marletta D, Tupac-Yupanqui I, Bordonaro S, Garcia D, Guastella AM, Criscione A, et al. Analysis of genetic diversity and the determination of relationships among western Mediterranean horse breeds using microsatellite markers. J Anim Breed Genet. 2006;123:315-25.

4. Blackburn HD, Paiva SR, Wildeus S, Getz W, Waldron D, Stobart R, et al. Genetic structure and diversity among sheep breeds in the United States: identification of the major gene pools. J Anim Sci. 2011;89:2336-48.

5. Zenger KR, Khatkar MS, Cavanagh JA, Hawken RJ, Raadsma HW. Genomewide genetic diversity of Holstein Friesian cattle reveals new insights into Australian and global population variability, including impact of selection. Anim Genet. 2007:38:7-14.

6. Lin BZ, Sasazaki S, Mannen H. Genetic diversity and structure in Bos taurus and Bos indicus populations analyzed by SNP markers. Anim Sci J. 2010;81:281-9.

7. Purfield DC, Berry DP, McParland S, Bradley DG. Runs of homozygosity and population history in cattle. BMC Genet. 2012;13:70.

8. Zhang L, Mousel MR, Wu X, Michal JJ, Zhou X, Ding B, et al. Genome-wide genetic diversity and differentially selected regions among Suffolk, Rambouillet, Columbia, Polypay, and Targhee sheep. PLoS One. 2013:8:e65942.

9. Flury C, Tapio M, Sonstegard T, Drögemüller C, Leeb T, Simianer H, et al. Effective population size of an indigenous Swiss cattle breed estimated from linkage disequilibrium. J Anim Breed Genet. 2010;127:339-47.

10. Toro MA, Caballero A. Characterization and conservation of genetic diversity in subdivided populations. Philos Trans R Soc Lond B Biol Sci. 2005;360:1367-78.

11. Crispim BA, Grisolia AB, Seno LO, Egito AA, Vargas Junior FM, Souza MR. Genetic diversity of locally adapted sheep from Pantanal region of Mato Grosso do Sul. Genet Mol Res. 2013;12:5458-66.

12. Hayes BJ, Visscher PM, McPartlan HC, Goddard ME. Novel multilocus measure of linkage disequilibrium to estimate past effective population size. Genome Res. 2003;13:635-43.

13. Tenesa A, Navarro P, Hayes BJ, Duffy DL, Clarke GM, Goddard ME, et al. Recent human effective population size estimated from linkage disequilibrium. Genome Res. 2007;17:520-6.

14. Wang J. Estimation of effective population sizes from data on genetic markers. Philos Trans R Soc Lond B Biol Sci. 2005;360:1395-409.

15. McKay SD, Schnabel RD, Murdoch BM, Matukumalli LK, Aerts J, Coppieters $W$, et al. Whole genome linkage disequilibrium maps in cattle. BMC Genet. 2007;8:74.
16. Espigolan R, Baldi F, Boligon AA, Souza FR, Gordo DG, Tonussi RL, et al. Study of whole genome linkage disequilibrium in Nellore cattle. BMC Genomics. 2013;14:305.

17. Porto-Neto LR, Kijas JW, Reverter A. The extent of linkage disequilibrium in beef cattle breeds using high-density SNP genotypes. Genet Sel Evol. 2014,46:22

18. Uimari P, Tapio M. Extent of linkage disequilibrium and effective population size in Finnish Landrace and Finnish Yorkshire pig breeds. J Anim Sci. 2011;89:609-14

19. Corbin LJ, Blott SC, Swinburne JE, Vaudin M, Bishop SC, Woolliams JA. Linkage disequilibrium and historical effective population size in the thoroughbred horse. Anim Genet. 2010;41:8-15.

20. Rao YS, Liang Y, Xia MN, Shen X, Du YJ, Luo CG, et al. Extent of linkage disequilibrium in wild and domestic chicken populations. Hereditas. 2008; 145:251-7.

21. Kijas JW, Porto-Neto L, Dominik S, Reverter A, Bunch R, McCulloch R, et al. Linkage disequilibrium over short physical distances measured in sheep using a high-density SNP chip. Anim Genet. 2014;45:754-7.

22. Meadows JRS, Chan EKF, Kijas JW. Linkage disequilibrium compared between five populations of domestic sheep. BMC Genet. 2008;9:61.

23. Garcia-Gamez E, Sahana G, Gutierrez-Gil B, Arranz JJ. Linkage disequilibrium and inbreeding estimation in Spanish Churra sheep. BMC Genet. 2012;13:43.

24. Gondro C, Porto-Neto LR, Lee SH. SNPQC-an R pipeline for quality control of illumina SNP genotyping array data. Anim Genet. 2014;45:758-61.

25. Browning BL, Browning SR. A unified approach to genotype imputation and haplotype-phase inference for large data sets of trios and unrelated individuals. Am J Hum Genet. 2009;84:210-23.

26. VanRaden PM. Efficient methods to compute genomic predictions. J Dairy Sci. 2008;91:4414-23.

27. Purcell S, Neale B, Todd-Brown K, Thomas L, Ferreira MA, Bender D, et al. PLINK: a tool set for whole-genome association and population-based linkage analyses. Am J Hum Genet. 2007:81:559-75.

28. Lencz T, Lambert C, DeRosse P, Burdick KE, Morgan TV, Kane JM, et al. Runs of homozygosity reveal highly penetrant recessive loci in schizophrenia. Proc Natl Acad Sci USA. 2007;104:19942-7.

29. Barrett JC, Fry B, Maller J, Daly MJ. Haploview: analysis and visualization of LD and haplotype maps. Bioinformatics. 2005;21:263-5.

30. Gabriel SB, Schaffner SF, Nguyen H, Moore JM, Roy J, Blumenstiel B, et al. The structure of haplotype blocks in the human genome. Science. 2002;296:2225-9.

31. Do C, Waples RS, Peel D, Macbeth GM, Tillett BJ, Ovenden JR. NeEstimator $\mathrm{V}$ : re-implementation of software for the estimation of contemporary effective population size (Ne) from genetic data. Mol Ecol Resour. 2014;14:209-14

32. Waples RS, Do C. Idne: a program for estimating effective population size from data on linkage disequilibrium. Mol Ecol Resour. 2008;8:753-6.

33. Yang J, Lee SH, Goddard ME, Visscher PM. GCTA: a tool for genome-wide complex trait analysis. Am J Hum Genet. 2011;88:76-82.

34. Meat Australian, Corporation Livestock. Handbook of Australian livestock. Sydney: Meat and Livestock Australia; 2000.

35. Hoze C, Fouilloux MN, Venot E, Guillaume F, Dassonneville R, Fritz S, et al. High-density marker imputation accuracy in sixteen French cattle breeds. Genet Sel Evol. 2013;45:33.

36. Stern JA, White SN, Meurs KM. Extent of linkage disequilibrium in large-breed dogs: chromosomal and breed variation. Mamm Genome. 2013;24:409-15.

37. Li MH, Stranden I, Kantanen J. Genetic diversity and pedigree analysis of the Finnsheep breed. J Anim Sci. 2009;87:1598-605

38. Boyles AL, Scott WK, Martin ER, Schmidt S, Li YJ, Ashley-Koch A, et al. Linkage disequilibrium inflates type I error rates in multipoint linkage analysis when parental genotypes are missing. Hum Hered. 2005;59:220-7.

39. McRae AF, McEwan JC, Dodds KG, Wilson T, Crawford AM, Slate J. Linkage disequilibrium in domestic sheep. Genetics. 2002;160:1113-22.

40. Kruglyak L. Prospects for whole-genome linkage disequilibrium mapping of common disease genes. Nat Genet. 1999;22:139-44.

41. Khatkar MS, Nicholas FW, Collins AR, Zenger KR, Al Cavanagh J, Barris W, et al. Extent of genome-wide linkage disequilibrium in Australian Holstein-Friesian cattle based on a high-density SNP panel. BMC Genomics. 2008:9:187. 
42. Bohmanova J, Sargolzaei M, Schenkel FS. Characteristics of linkage disequilibrium in North American Holsteins. BMC Genomics. 2010;11:421.

43. Du FX, Clutter AC, Lohuis MM. Characterizing linkage disequilibrium in pig populations. Int J Biol Sci. 2007;3:166-78.

44. Badke YM, Bates RO, Ernst CW, Schwab C, Steibel JP. Estimation of linkage disequilibrium in four US pig breeds. BMC Genomics. 2012;13:24.

45. Lindblad-Toh K, Wade CM, Mikkelsen TS, Karlsson EK, Jaffe DB, Kamal M, et al. Genome sequence, comparative analysis and haplotype structure of the domestic dog. Nature. 2005;438:803-19.

46. Hinds DA, Stuve LL, Nilsen GB, Halperin E, Eskin E, Ballinger DG, et al. Whole-genome patterns of common DNA variation in three human populations. Science. 2005;307:1072-9.

47. Qanbari S, Pimentel ECG, Tetens J, Thaller G, Lichtner P, Sharifi AR, et al. The pattern of linkage disequilibrium in German Holstein cattle. Anim Genet. 2010;41:346-56.

48. Porto-Neto LR, Lee SH, Sonstegard TS, Van Tassell CP, Lee HK, Gibson $J P$, et al. Genome-wide detection of signatures of selection in Korean Hanwoo cattle. Anim Genet. 2014:45:180-90.
49. Johnston SE, McEwan JC, Pickering NK, Kijas JW, Beraldi D, Pilkington JG, et al. Genome-wide association mapping identifies the genetic basis of discrete and quantitative variation in sexual weaponry in a wild sheep population. Mol Ecol. 2011;20:2555-66.

50. Gautier M, Naves M. Footprints of selection in the ancestral admixture of a New World Creole cattle breed. Mol Ecol. 2011;20:3128-43.

51. Beraldi D, McRae AF, Gratten J, Slate J, Visscher PM, Pemberton JM. Development of a linkage map and mapping of phenotypic polymorphisms in a free-living population of Soay sheep (Ovis aries). Genetics. 2006;173:1521-37.

52. Johnston SE, Beraldi D, McRae AF, Pemberton JM, Slate J. Horn type and horn length genes map to the same chromosomal region in Soay sheep. Heredity (Edinb). 2010;104:196-205.

53. Han J, Kraft P, Nan H, Guo Q, Chen C, Qureshi A, et al. A genome-wide association study identifies novel alleles associated with hair color and skin pigmentation. PLoS Genet. 2008;4:e1000074.

\section{Submit your next manuscript to BioMed Central and take full advantage of:}

- Convenient online submission

- Thorough peer review

- No space constraints or color figure charges

- Immediate publication on acceptance

- Inclusion in PubMed, CAS, Scopus and Google Scholar

- Research which is freely available for redistribution

Submit your manuscript at www.biomedcentral.com/submit
C Biomed Central 